\section{Antibiotic induced changes to mitochondria result in potential contributions to carcinogenesis, heart pathologies, other medical conditions and ecosystem risks}

\author{
Jorma Jyrkkanen*
}

British Columbia, Canada

\section{Abstract}

With the discovery by Calghatgi (2013) that three common antibiotics (Abs) increased mitochondrial reactive oxygen (ROS) and lipid peroxide (LP) and depleted their natural absorbant glutathione led me to investigate further the potential impacts of these genotoxic substances on carcinogenesis. The range of impacts on mitochondria and cellular DNA varied by antibiotic to those consistent with known prior contributions to carcinogenesis. Specific cancers probably increased by these changes were HCC, RCC (KCC), CRC, cancer of the esophagus. Tumor suppressor gene mutations resulting from LP were noteworthy in this regard and mutations induced in CRC were consistent with those found in carcinogenesis of CRC. In addition depression of short chain fatty acids in microbiomes were found which depress the immune system increasing risk of all cancers. Many cancers were increased according to epidemiological studies linking Abs with elevated odds ratios, with one concern in particular, fatal breast cancer. The impact of loss of functionality of the mitochondria was also linked to depression of the citric acid cycle and therefore ATP which deflected metabolism to glycolysis, the Warburg mechanism also increasing risk of all cancers, favoured by cancer cells. In conclusion, some portion of many cancer types are probably increased in likelihood by number, type and frequency of Abs treatment and chronic residue exposure which varies from individual to individual. This led me to propose a three pronged carcinogenesis mechanism for Abs. 1. Cancer critical mutations 2. Immune depression 3. loss of mitochondrial functionality leading to Warburg effects. Damage to mitochondria were also noted by common pesticides tested in China and cancer associations were also found for many pesticides supporting a similar contributory etiology. Heart health concerns were raised by these findings because of the myriad mitochondria in the heart and because of long term reliability needs. Studies suggesting hearts were affected by Abs and pesticide exposure were presented. Because of their geographical ubiquitousness and the huge range of diseases associated with mitochondrial dysfunction, antibiotics and pesticides and bacteriocidal biocides are of concern for biodiversity and life in general. I propose research steps to evaluate Abs safety and suggest directions for further research and make suggestions on ways to ameliorate Abs toxicity.
More Information

*Address for Correspondence: Jorma Jyrkkanen, British Columbia, Canada, Tel:1-250-859-5330; Email: jormabio@hotmail.com

Submitted: 17 August 2020

Approved: 01 October 2020

Published: 02 October 2020

How to cite this article: Jyrkkanen J. Antibiotic induced changes to mitochondria result in potential contributions to carcinogenesis, heart pathologies, other medical conditions and ecosystem risks. J Cardiol Cardiovasc Med. 2020; 5: 163-171.

DOI: 10.29328/journal.jccm.1001104

Copyright: @ 2020 Jyrkkanen J. This is an open access article distributed under the Creative Commons Attribution License, which permits unrestricted use, distribution, and reproduction in any medium, provided the original work is properly cited.

Keywords: Antibiotic; Mitochondria; DNA damage; P53 tumor suppressor cene; Mutp53; ROS; Lipid peroxide; Cell perforation; Cell rupture; Oxidative phosphorylation; Cancer; Carcinogenesis; Glycolysis; Warburg effect; Microbiome; Dysbiosis; Immune suppression; Pesticides; Mechanism; Clastogenic; Epigenetic silencing; Microrna; DNA; Heart

Check for updates

OPEN ACCESS

\section{Introduction}

Antibiotics kill or slow the growth of bacteria or interfere in their reproduction. The mitochondria, an ancient alphaproteobacteria [Rickettseae] that has becomean endosymbiont in higher life forms with critical functions, response to them has been found to be a decrease of beneficial antioxidant glutathione, increased reactive oxygen, increased harmful lipid peroxide, possible DNA damage and mutations in tumour suppressor genes increasing cancer risk, possible inability to reproduce, possible cell perforation and or rupture. Some antibiotics have been shown in the past to be clastogenic. These types of responses have broad biochemical and health implications. They could lead to carcinogenisis, microbiome dysbiosis with resulting immune system depression and or loss of oxidative phosphorylation (OP) favouring glycolysis metabolism which is also the favoured method for cancer cells. Changes could enhance the Warburg effect favouring cancers. P53 genes may be turned off epigenetically at the DNA. Defective mitochondria have been implicated in over 
200 medical conditions. In addition a big unknown is the relationship between which biocides may epigenetically shut down critical genes found with each particular kind of cancer. Clinical and epidemiological evidence supports the conclusion that some antibiotics are carcinogens, others promote cancers and cancer risk increases with frequency and type. Microbiome dysbiosis and immune depression risk is increased. While exposure may not complete all the steps to cancer it may contribute important mutations along the way. Other life time exposures can can complete the process. Chinese researchers recently found that a high proportion of common pesticides ruptured mitochondria like some antibiotics. Individuals who were exposed to pesticides were more than twice as likely overall to have conditions like heart disease, heart failure or an irregular rapid heartbeat known as atrial fibrillation [1]. It can be inferred that ruptured mitochondria from antibiotics would lead to similar coronary pathologies. There is reason to suspect that all Eukaryotes are subject to pathological impacts. The mitochondria enabled multicellular evolution to higher forms of life and is now under attack worldwide by anthropogenic biocide pollution. More research is needed to determine which of all biocides is mitochondria friendly, enables them to be fully functional without mutations, prior to regulatory approvals. I propose an antibiotic mitochondria carcinogenesis mechanism. For a general overview of impacts of antibiotics on total general physiology and health of ecosystems including plants see Wang, Xu et al. [2]. A criticism of some findings is that people with cancer are more prone to infections and this can account for much of the association of antibiotics with cancer [3]. However the finding of immune compromise is consistent with antibiotic induced microbiome associated dysbiosis.

\section{Mitochondrial job and creation of toxic mix by antibiotic}

Mitochondria, a primitive endosymbiotic bacteria, related to extant SARII marine bacteria and Rickettsias, in eukaryotes is responsible for OP resulting in ATP and NAD production for energy. When exposed to clinically equivalent doses of antibiotics that target bacteria (cipromycin, ampicillin, kanamycin), exhibited a decline in glutathione titre, an increase in reactive oxygen (ROS) and an increase in lipid peroxide with damage to DNA and potential mitochondrial rupture [4]. Tetracyclines used for humans and livestock have also been linked to mitochondrial genetic damage [5]. Some antibiotics have been found to be break chromosomes [6].

Modes of action of antibiotics on mitochondria and microbiome

1. quinolones- commonly prescribed antibacterial organofluorine compounds which act by inhibition of bacterial DNA synthesis and result in rapid cell death [7]. This group contains ofloxacin, norfloxacin (noroxin), ciprofloxacin (Cipro), moxyfloxacin (Avelox). Expectation is to obstruct mitochondrial replication. Norfloxacin demonstrated a linear
antibiotic-DNA mutation rate, compromised DNA oxidative damage repair and post replicative mismatch repair [8]. They could be expected to do similar collateral damage to mitochondria and to members of the human microbiome.

2. aminoglycosides-ex gentamicin, amicasin which create holes in the outer cell wall of bacteria suggesting mitochondria and the microbiome might be at risk of similar damage [9]. Damage to lipid membranes can be expected. Lipid membranes have wide distribution in both microbes and other animals including humans.

3. $\boldsymbol{\beta}$-lactams or penicillin derivatives such as cephalosporins, monobactams, carbapenems, carbacephems inhibit cell wall synthesis in bacteria and by inference inhibit cell wall synthesis in mitochondria during division and repair and microbiomes thereby obstructing microbial reproduction. Penicillamine is listed as a 'developmental' in California Proposition 65.

4. Tetracyclines-used on cattle and humans and possibly acquired secondarily as dietary residues may affect mitochondria because they specifically target Rickettsias a probable evolutionary ancestor $[2,10.11]$.

\section{Anthracyclines-result in clastogenicity [6]}

Harmful impact of liberated substances on DNA, P53 tumour suppressor gene, mutagenicity and known effects in other cancers

Glutathione is an antioxidant that soaks up ROS and is essential for many neurological and other body functions. Glutathione is capable of preventing damage to important cellular components caused by reactive oxygen species such as free radicals, peroxides, lipid peroxides, and heavy metals. Genomic instability occurs in myeloid malignancies with increased reactive oxygen species ROS, DNA double strand breaks (DSBs) and error-prone repair [12].

\section{ROS linked to many cancers by oxidative DNA damage}

"numerous studies have shown generation of reactive oxygen species (ROS) that can cause oxidative damage of DNA. This is a well-known mechanism in carcinogenesis for many agents" [13].

Excessive levels of ROS accumulation due to altered equilibrium between ROS and antioxidants may lead to different kinds of diseases such as atherosclerosis, diabetes, neurodegeneration, and cancer including CRC. It is widely known that ROS-induced DNA damages and genetic mutations are critical causes of cancers including CRC. The main intracellular DNA lesions caused by ROS are single and double strand DNA breaks, and the common genetic mutations include p53, KRAS, APC, and BRAF mutations often seen in CRC's. For example, a direct relation among oxidative stress, DNA damage and elevated frequency of p53 mutation in CRC 
has been observed. Most extensively studied endogenous DNA damage by ROS is the formation of 8-oxo-7,8-dihydro2'-deoxyguanosine (8-oxodG). As the biomarker of oxidative stress, 8-oxodG level is higher in colorectal tumors than in normal mucosa. Mitochondrial DNA is particularly prone to be oxidatively damaged and is more meaningful in colorectal carcinogenesis [14].

I would expect antibiotic induced drop in antioxidant glutathione to contribute to such an altered equilibrium and assay for 8-oxodG post antibiotic treatment might be a good indicator of antibiotic carcinogenic potential as well as looking for deficits of ATP, an indicator that metabolism has switched to cancer cell loving glycolysis from pyruvate metabolism.

\section{Lipid peroxide associated cancers}

Besides being generated by mitochondria exposed to antibiotics, lipid peroxide is also increased with analgesics like aspirin (though some studies show it reduces LP) and NSAIDS naproxin, indomethacin and diclofenac, being male, among hypertensives, diabetics, smokers, oophorectomized and pregnant women especially with eclampsia and preeclampsia [15]. Ochratoxin a mycotoxin found in cereals and grains also increases LP. These mutiple sources need assaying when making links to antibiotics impact on mitochondria.

Lipid peroxide has been linked to esophageal carcinogenesis [16] and to red meat and treated meat colon carcinogenesis [17]. The major lipid peroxidation product, trans-4-hydroxy-2-nonenal, preferentially forms DNA adducts at codon 249 of human p53 gene, a unique mutational hotspot in hepatocellular carcinoma [18]. In a seemingly unrelated exposure from afltoxin researchers report an increased frequency of loss of the Hae III allele and base $\mathrm{G}$ mutation on p53 gene at codon 249 where it is mutated to $C$ [19]. Why this matters is because this same P53 gene locus is linked to HPV cervical cancers from a study done on Kenyan women [20]. HPV cancers are associated with genital, anal and oral tissues. Antibiotics production of lipid peroxide and its metabolites can also mutate this gene locus and that is found with HCC.

Lipid peroxidation has been proposed as a mechanism for renal cell carcinoma RCC [15]. Based on their work I added my comments. Lipid peroxide (LP) degrades into mutagens that target tumor suppressor gene p53 and may alter functionality of other tumor suppressor genes like VHL specifically linked to hereditary RCC and is postulated by me and the latter authors to be a carcinogen linked to renal cell carcinoma. I phrase this as a question. Lipid peroxide is generated by antibiotics attacking mitochondria which then release it into the tissue environment and may even rupture cells in the process. I recommend an investigation to study additionally antibiotic history in regards to VHL depressed kidney cancer. The placenta is the main source of LP in pregnant women. Look also at Aspergillis and ochratoxin A as a mutagen for the TSG genes involved. The following article hints at LP causality.
Fumonisims, a fungus in corn and other grains is linked to kidney cancer and is possibly acting through mutation of the p53 gene. P53 overexpression has been correlated with increased RCC. Inactivation of the VHL TSG is responsible for polycystic kidney disease and for renal cell carcinoma of the hereditary VHL cancer syndrome and for the majority of sporadic renal cell carcinomas.

Protectively, polyphenolics in red wine are postulated to soak up the lipid peroxides and reduce RCC risk. Estrogens especially 2-hydroxyestradiol, mannitol, SOD and vitamin E are all LP sponges along with the mitochondrial glutathione.

This suggests antibiotics are a cofactor in carcinogenesis of several if not multiple cancers via this same p53 locus 249 mutation's contribution or by lipid peroxide contribution and ROS contributions to reduce TSG DNA repair and function. Lipid peroxide metabolite hydroxy-2-nonenal is also found in red meat and treated meat carcinogenesis. It is safe to conclude that antibiotics are one cause of or major contributing factor to hepatocelluar carcinoma and are also potentially involved in colon carcinogenesis. In CRC, the commonest lipid peroxidation products are MDA and HNE, the levels of which in the CRC tissue are significantly increased with clinical staging [21]. CRC and RCC are likely to be cancers potentially associated with antibiotic mitochondrial disruption.

\section{P53 changes associated with warburg effect}

Possible P53 gene upregulation (PUMA->WTP53) may lead to the Warburg effect favouring cancer [22]. Proximicins A, B, and C-antitumor furan analogues of netropsin from the Marine Actinomycete verrucosispora induce upregulation of p53 though I am not certain this is the same effect as PUMA [23]. Mutated P53 actually becomes the mutP53 guardian of cancer cells [24].

\section{Normal function of p53 blocked by loss of mitochondria through damage or rupture}

Tumor suppressor p53 plays a central role in tumor prevention. As a transcription factor, p53 mainly exerts its function in tumor suppression through its transcriptional regulation of its target genes to initiate various cellular responses.

Cell cycle arrest, apoptosis and senescence are most wellunderstood functions of p53, and are traditionally accepted as the major mechanisms for p53 in tumor suppression. Recent studies have revealed a novel function of p53 in regulation of cellular metabolism. p53 regulates mitochondrial oxidative phosphorylation, glycolysis, glutamine metabolism, lipid metabolism, and antioxidant defense. Through the regulation of these metabolic processes, p53 maintains the homeostasis of cellular metabolism and redox balance in cells, which contributes significantly to the role of p53 as a tumor suppressor [25]. 
P53 Cell guardianship and critical OP obviously cannot happen if the mitochondria is ruptured or defective or if the p53 gene has been mutated, silenced or sequestered to assist cancer cells. With diminished OP, Warburg effects will increase and cancer cells will be given a boost. This might well be a serious collateral impact of antibiotics.

\section{Antibiotics render the immune system less effective in infection}

Researchers reporting in Frontiers in Microbiology found that short chain fatty acids (SCFA) from resident bacteria were important in protecting the immune system, and inflammation control. Both of these side effects have important ramifications for prevention of cancer initiation. Antibiotics diminished resident bacteria carrying out this role and supplemental SCFA were not effective in ameliorating the effect.

Dysbiosis of resident microbes is unequivocally associated with immune-related disorders and opportunistic and pathogenic infections which can themselves set the stage for cancer [26]. If potentially carcinogenic microbes Helicobacter pylori, Streptococcus bovis, Salmonella typhae, Fusobacterium, Chlamydophyla, Bartonella or Caries bacteria or any carcinogenic viruses such as EBV, HPV, alpha-HPV, beta-HPV, HHV, HBV, HVC, KSHV and possibly retroviruses or Schistosomes and liver flukes facilitated by [27] depressed immune systems proliferate as a consequence this can lead to increased incidence of cancers especially the viral cancers which do not respond to antibiotics but will take advantage of a depressed immune system.

Along this line there has been an increase in oropharyngeal HPV cancers in Canadian men [28]. The depressed immune system may also lessen the bodies ability to kill cancerous cells regardless of their etiological origins. Another of the consequences of antibiotic use is development of antibiotic resistance. One of the carcinogenic bacteria, H. Pylori is an example [29] the consequence of which may lead to an increase in stomach cancers in developed countries unless we can come up with new more effective mitochondria friendly antibiotics.

\section{The evidence of carcinogenesis from research}

Seeing that these changes were consistent with steps found in carcinogenesis [30] I asked the question, what is the clinical and epidemiological evidence that antibiotics increase the risk of cancer? It appears others have also addressed this question [31,32], [antibiotic use predicts an increase in the risk of cancer]; I reproduce Kilkkinen's results because they speak to the range of cancers brought under suspicion.

"The use of antibiotics was associated with an increased risk of cancer; for categories of increasing antibiotic use (0-1, 2-5 and \# 6 prescriptions), RRs (95\% CIs) were 1.0 (reference), 1.27 (1.26-1.29) and 1.37 (1.34-1.40). The association was found both in men (RR for comparison of lowest and highest exposure group 1.47, 95\% CI 1.42-1.53) and women (RR 1.31, 95\% CI 1.28-1.35). The most common cancers i.e. prostate, breast, lung and colon comprised half of all cancer cases; RR $(95 \% \mathrm{CI})$ was $1.39(1.31-1.48)$ for prostate, $1.14(1.09-1.20)$ for breast, 1.79 (1.67-1.92) for lung, and 1.15 (1.04-1.26) for colon cancer. RRs for other primary sites varied between $0.90(0.76-1.05)$ for ovary and $2.60(1.60-4.20)$ for endocrine gland cancers. In addition to endocrine gland and liver cancers, the risk of non-melanoma skin, duodenum, pancreas, kidney, bladder, male genitals (excluding prostate) and thyroid cancers as well as myeloma and leukemia was more than 1.5 times higher among participants with 6 or more antibiotic prescriptions compared with the lowest exposure group. Restricting analyses to participants with 5 or more years follow-up did not produce significantly different results from those covering the entire study population (RR for the comparison of lowest and highest exposure group 1.37, 95\% CI 1.34-1.40). Similar results were obtained when the data were stratified according to age (data not shown). We also observed an increased risk of death due to cancer with use of antibiotics (RR 1.33, 95\% CI 1.28-1.38). There was a similar tendency for an increased cancer risk with annual antibiotic use (table is available from authors by request). Compared with non-users of antibiotics, RRs $(95 \% \mathrm{CI})$ for 1 year, 2 and 3 years of use were 1.33 (95\% CI 1.32-1.35), 1.40 (1.38-1.42) and 1.46 (1.43-1.49), respectively. RRs (95\% CI) for 3 years of use for different primary sites varied from 0.99 (0.86-1.14) for ovary to 1.81 (95\% CI 1.62-2.02) for non-melanoma skin cancers and was $1.21(1.15-1.26)$ for breast and 1.57 (1.491.66) for prostate cancers."

Tim Newman 2017 [33] [antibiotics may increase the risk of bowel cancer]; Millipore-Sigma 516104 [34]. [penicillin/streptomycin/amphotericin-harmful, teratogenic and carcinogenic]). Velicer et al. [35] found prolonged use of antibiotic increased risk of fatal breast cancer. This has broad global ramifications because of the chronic long term exposure of antibiotic residues in diet from treated foods such as beef, pork, poultry and farmed fish and sea food products. In a new epidemiological study 'intakes of dairy calories and dairy milk were associated with BC hazard ratios (HRs) of 1.22 [95\% confidence interval (CI): 1.05-1.40] and 1.50 (95\% CI 1.22-1.84)' [36].

These results are deeply troubling despite any experimental difficulties because they almost unanimously point in the same direction to increasing carcinogenicity and the huge global populations exposed to residues. Zhang et al. [3] offered criticisms of association studies and the reader is encouraged to weigh them against the evidence presented here. My rebuttle is that the above findings are also consistent with reactive oxygen DNA adduct mutations' range of cancers, defective tumor suppressor genes and Warburg effects from mitochondrial OP knockdown and antibiotic induced dysbiosis induced immune compromise. 


\section{IARC anomaly discussion}

The problem in developed countries is that use a lot of antibiotics is that infection related cancers are lower than in the Third World [cf. 7.4\% versus 22.9\%] showing that they are protective for these but overall reported in 2019 in Canada for example is that cancer incidence reported is $50 \%$ [37]. This suggests carcinogen and life style related cancers (see Proposition 65 list) and perhaps population of age classes contribute to the high rate. This anomaly suggests cumulative mutations and other mechanisms such as I am investigating and exposures are setting the stage for later cancer illness. That we have long insidious exposure to mutation inducing carcinogens and endocrine disruptors is confirmed by my review of pesticide and chemical carcinogens in mothers breast milk [38].

\section{CRC cancers increasing in Canada}

How safe and contributory to cancer are antibiotics? This can be addressed considerably by the experiments I propose at the end of this article. Colorectal cancers (CRC) are very informative in this story. CRC incidence increased exclusively in young adults in nine high-income countries spanning three continents, potentially signalling changes in early-life exposures that influence large bowel carcinogenesis [39] and a fail for antibiotics for this cancer and microbiome dysbiosis may play a pivotal role. Another intriguing possibility is inherited epigenetic markers from parental exposures. Alcohol and obesity are confounding factors for CRC etiology with interaction effects [40]. Downregulation of tumor suppressor gene TUSC3 facilitates proliferation of colon cancer CRC. Its absence or dysfunction of expression after exposure to chemicals and drugs can give a prognosis of chemicals safety [41].

\section{Antibiotics change tissue environment to favour cancer metabolism by the warburg effect}

In addition, one of the antibiotics classes tested was linked to a decline in pyruvate, the feed stock for the citric acid cycle and ATP and NAD production or OP. With the loss or reduction of OP the default respiration glycolysis increases dominance. This is called the Warburg Effect which cancer cells have been shown to prefer in which they employ glycolysis instead of OP for their energy and this may result from defects in mitochondria(https://en.wikipedia.org/wiki/Warburg effect_(oncology)).

It can be expected that this ideal environment for glycolysis favouring cancer cells will be the norm whenever and wherever mitochondria are damaged or ruptured as they are with these antibiotics tested and with common pesticides and if pyruvate is specifically diminished.

Increasing mitochondrial reproduction should reverse this process by restoring OP, replacing the Warburg effect, and that is exactly what is found [42]. This finding is strong confirmation of the carcinogenic effect of losing mitochondria and their function. Aerobic exercise suggests itself to me as a way to build up mitochondria and fight carcinogenesis via this mechanism.

My Proposed Antibiotic Mitochondrial Carcinogenicity Mechanism AMCM

\section{Requirements for carcinogenesis contribution}

Induce mitochondrial malfunction, damage or rupture-YES; prevent mitochondria from faithful reproduction in quality and quantity-Probable; cause mutations and genetic material damage-YES; interfere with tumour suppressor genes-YES; create microbiome dysbiosis-YES; harm the immune systemYES; increase the Warburg effect-YES; statistically significant associations-Probable.

\section{AMCM}

1. Reactive oxygen linked to oxidative mutations of DNA found in many cancers and Lipid peroxide induces P53 mutations and mutated P53 no longer repairs DNA and may in fact assist cancer cells and probably the primary cause of some portion of hepatocelluar carcinomas

2. Glutathione deficiency increases toxicity of many metabolites it normally neutralizes

3. Obstruct mitochondrial and microbiome lipid membrane integrity and mitochondrial replication leading to $\mathrm{OP}$ reduction and increasing microbiome dysbiosis

4. Reduction of OP and mutant P53 increase Warburg Effect favouring cancer cells glycolysis providing an advantage to cancer cells

5. Antibiotic induced microbiome dysbiosis immune compromise decreasing efficacy of subjects cancer immune defense mechanisms

Further research suggested by these studies includes testing all antibiotics for their mitochondrial impacts.

\section{Related mitochondrial stressors and potential ramifica- tions}

These findings also raise the question are there pesticides with similar consequences? There are intriguing findings in China. 9 Common pesticides tested induce morphological changes of mitochondria at low concentrations. Paraquat, rotenone, chlorpyrifos, pendimethalin, endosulfan, fenpyroximate and tebufenpyrad induced mitochondria fragmentation. Furthermore, some of them (paraquat, rotenone, chlorpyrifos, fenpyroximate and tebufenpyrad) caused a significant dose-dependent decrease of intracellular ATP suggesting increased risk of Warburg syndrome because ATP is a proxy for OP integrity. Interestingly, these pesticides which induce mitochondria dysfunction also inhibit $26 \mathrm{~S}$ 
and 20S proteasome activity [43] which suggests to me we should be looking at antibiotics and proteasome homeostasis because of its required integrity for health. These results in turn raise the obvious question, are the consequences similar in terms of potential long term carcinogenicity? The answer is yes [44] in which they state that "Chemicals in every major functional class of pesticides including insecticides, herbicide, fungicides, and fumigants have been observed to have significant associations with an array of cancer sites".

\section{Biocide-mitochondrial effects on heart function}

Another interesting question. The heart muscle is full of mitochondria. Do antibiotics and pesticides affect the hearts mitochondria and if so in what way and for how long? I would expect this heart loss of OP combined with ROS and increased peroxides to lead to a condition like chronic fatigue and possibly compromised coronary function. Azithromycin induced increased deaths in patients with prior coronary issues according to study authors Wayne A. Ray, Ph.D. and C. Michael Stein, M.B., Ch.B., and Dan May 17 May 2012. John R. Giudicessi et al. 2013 [45,46] also found increased risk of sudden death from Azithromycin. Azithromycin is a macrolide which prevents bacteria from growing by interfering with their protein synthesis. How this might be linked to mitochondrial protein production needs to be examined. A UBC study found a $2.4 \mathrm{X}$ increased risk of mitral valve regurgitation in fluoroquinolone users [47]. This is an area needing more research and review. FDA [48] issued a warning that some antibiotics used for URI's and urinary infections can cause aortic rupture and prescriptions to people at risk is contraindicated.

\section{Lifetime cumulative augmentation}

Cumulative antibiotic for clinical treatment exposures are unwittingly augmented by chronic low level residues of other antibiotics from dietary sources like poultry, beef, farmed fish and pork and may not immediately cause a cancer but may contribute to the conditions for one to occur at a later date by facilitating entry of carcinogenic infectious agents. Other mutagens and carcinogen residues (See California Proposition 65 List), radiation, chemical and pesticide residues and immune decline with age can complete the cancer induction.

Calghatgi (1973) suggests that deleterious effects of bactericidal antibiotics were alleviated in cell culture and in mice by the administration of the antioxidant N-acetyl-lcysteine or prevented by preferential use of bacteriostatic antibiotics. Is this sufficient to eliminate the microbiome dysbiosis immune depression effects and does it work for all antibiotics including tetracycline which specifically targets relatives of mitochondria in humans? This needs critical examination because of the enormous populations exposed.

\section{An evolution approach enables these extrapolations}

These findings in normal mitochondria of their stress response to antibiotic biocides is consistent with their evolutionary origin from Rickettseae alpha-proteobacteria and are linked to biochemical pathways already shown linked to carcinogenesis and confirmed in the literature. Another probably most definitive path to investigate the carcinogenicity of antibiotics is to run epigenetic profiles on them to determine of they are able to turnoff genes found turned off in DNA and microRNA in cancers [49,50]. Each antibiotic and mitochondria rupturing pesticide should be put through trials to look for P53 upregulation, mutation (mutP53) and epigenetic silencing in mice and rats at ppb resolutions. Calghatgi tests should be included on all antibiotics and pesticides that rupture mitochondria at ppb resolutions as well as testing for missense mutations, characteristic of p53 mutations associated with carcinogenesis [51] and of course the CRC risk indicator assay pre and post antibiotic treatment for [8-oxodG] titre. A simple test to determine Warburg potential would be to compare pre and post antibiotic treatment intracellular ATP titre. Uncontacted Tribes with infection linked cancers might also be good controls for p53 mutations, p53 upregulations, p53 epigenetic silencings but some of them would of course have been exposed to plants that have traditional medical effects [52] and their sample size would be small. Another potential cell guardian to assess for antibiotic related mutations, epigenetic silencing is the suspected tumor suppressor trichoplein/mitostatin (TpMs) which inhibits mitofusin-2 and hence mitochondrial associated membrane formation, but is downregulated or mutated in a number of types of cancer [53].

\section{Challange to my fundamental thesis, Wallace 2012 [54]}

Wallace opens with cancer needs functional mitochondria to prosper. This seems to imply disaster for my above hypothesis. However in further reading I find his conclusions strongly supportive. My response to his work is this. How do his findings speak to my antibiotic-mitochondrial knockdowncancer hypothesis? The glutathione, ROS, lipid peroxide DNA mutagenicity, microbiome dysbiosis are part of my answer. He also [supportively] states "mitochondrial reactive oxygen species (ROS)........altering the activities of transcription factors such as HIF1 $\alpha$ and FOS-JUN to change gene expression and stimulate cancer cell proliferation." Moreover he adds "Cancer cell ROS production inactivates caveolin 1 in adjacent stromal fibroblasts. This increases mitophagy, reduces mitochondrial function and increases lactate production in these fibroblasts. Secreted stromal cell lactate then fuels cancer cell oxidative metabolism, which drives tumour growth and proliferation. This is known as the 'reverse Warburg effect'. Its clear that one analysis needed to determine antibiotic/biocide/selected pesticide carcinogenicity is to measure the cancer cells mitochondrial ROS and lipid peroxide output in response to biocide use. I expect cancer cell mitochondria to respond the same as normal cellular mitochondria with low glutathione, increased ROS and lipid peroxide. This is an easy test. A careful read shows his work is supportive of my hypothesis. 


\section{Follow up research}

To resolve the safety of antibiotics (Abs) regarding their potential impact on mitochondrial impacts, I have arrived a number of research questions which can help steer our understanding and antibiotic futures. Independent academic institutional involvement is preferred so that vested interests don't cloud the results.

1. How large and persistent is the glutathione decrease, ROS and lipid peroxide increase post antibiotic treatment for each antibiotic if any?

2. What effect do Abs have on future reproduction, integrity and populations of mitochondria in heart muscle.

3. Need to survey and list genetic mutations and epigenetic alteration of cancer gene expression and especially cancer gene silencing post Abs treatment in DNA and microRNA.

4. Assess dysbiosis linked impacts on immune system post Abs treatment and duration of the effect.

5. Do a full analysis of coronary function and mitochondrial health post Abs for each Abs.

6. Broad survey of Abs clastogenicity with complete description.

7. Which Abs rupture mitochondria and or cells and at what concentrations?

8. Assay 8-oxodG post Abs treatment to determine potential carcinogenicity?

9. Do any Abs impact on promotion of hepatocellular carcinoma and CRC?

10. Assay tumor suppressor epigenetic gene up-regulation, down-regulation, silencings and mutations and their loci for P53 and TUSC and TpMs.

11. Conduct a population study for Abs usage and Breast Cancer with proper controls and examine BC associated genes [55] for mutations or epigenetic silencings post Abs treatments and relationship to exposure history.

12. Assess OP and glycolysis potential of each Abs to determine potential for Warburg effect.

13. Abs history study of HPV oropharyngeal cancers in men in developed countries.

\section{Abs impact on proteasome activity.}

15. Assay Abs for reverse Warburg activity for $\mathrm{H} 1 \mathrm{~F}(\mathrm{alpha})$ and FOS-JUN which stimulate cancer cell proliferation.

16. Assay Abs treatment for caveolin 1 in adjacent stromal fibroblasts which increase mitophagy and lactate in fibroblasts driving tumor growth and proliferation.
17. Routine clinical assay for interstitial ATP titre recovery once antibiotic use is discontinued to see if the Warburg effect if initiated on application has been neutralized.

I think this would clarify the scale and degree of impact of antibiotics and point to areas needing remedies. Many other diseases are linked to mitochondrial alterations. The reader is referred to Salvatore diMauro and Darryl C De Vivo book Diseases of Mitochondrial Metabolism. Basic Neurochemistry: Molecular, Cellular and Medical Aspects. 6th edition. Siegel GJ, Agranoff BW, Albers RW, et al. [39], editors. Philadelphia: Lippincott-Raven; 1999. Salvatore diMauro and Darryl C De Vivo.

The most urgent in the light of growing antibiotic resistance are these two critical health questions, ie mitochondrial health relative to heart health and carcinogenicity contributions.

\section{References}

1. Lisa Rapaport. 2019. Health News January 9, 2019 / 3:30 PM/Reuters.

2. Wang X, Dongreol R, Johan A. Antibiotic use and abuse: A threat to mitochondria and chloroplasts with impact on research, health, and environment. Bioessays. 2016. 37: 1045-1053.

PubMed: https://www.ncbi.nlm.nih.gov/pmc/articles/PMC4698130/

3. Zhang $\mathrm{H}$, Rodríguez LAG, Hernández-Díaz S.. Antibiotic Use and the Risk of Lung Cancer. Cancer Epidemiol Biomarkers Prev. 2008; 17: 1308-1315.

PubMed: https://pubmed.ncbi.nlm.nih.gov/18544646/

4. Sameer C. Bactericidal antibiotics induce mitochondrial dysfunction and oxidative damage in mammalian cells. Sci Transl Med. 2013; 5: 192 ra85.

PubMed: https://pubmed.ncbi.nlm.nih.gov/23825301/

5. Granados-Chinchilla F, Rodríguez C. Tetracyclines in Food and Feeding stuffs: From Regulation to Analytical Methods, Bacterial Resistance, and Environmental and Health Implications. J Analytical Methods Chem. 2017: 1315497.

PubMed: https://pubmed.ncbi.nlm.nih.gov/28168081/

6. Nersessian AK, Zilfian Vrez N, Koumkoumadjian VA. Fanardjian. Inhibitory effect of rat immunization with tularemia vaccine on the in vivo clastogenicity of 4 anthracycline antibiotics. Mutation Research. 1991; 260: 215-218.

7. Aldre KJ, Kerns RJ, Neil O. Mechanism of Quinolone Action and Resistance. Biochemistry. 2014: 53: 1585-1574.

PubMed: https://pubmed.ncbi.nlm.nih.gov/24576155/

8. Long H, Miller SF, Strauss C, Zhao C, Cheng L, et al. Antibiotic treatment enhances the genome-wide mutation rate of target cells. PNAS 2016; 113: E2498-E2505. https://www.ncbi.nlm.nih.gov/pmc/articles/ PMC4983809/ Gonzalez L, Jianne P. Spencer. Aminoglycosides: A Practical Review Am Fam Physician. 1998; 15; 58:1811-1820. PubMed: https://pubmed.ncbi.nlm.nih.gov/9835856/

9. Emelyanov VV. Evolutionary relationship of Rickettsiae and mitochondria. 2001; 501: 11-18.

PubMed: https://pubmed.ncbi.nlm.nih.gov/11457448/

10. Moullan N, et al. Tetracyclines Disturb Mitochondrial Function across Eukaryotic Models: A Call for Caution in Biomedical Research." Cell reports. 2015; 10: 1681-1691.

PubMed: https://pubmed.ncbi.nlm.nih.gov/25772356/

11. Sallmyr A, Feyruz JF, Rassool V. Genomic instability in myeloid malignancies: Increased reactive oxygen species (ROS), DNA double strand breaks (DSBs) and error-prone repair. Cancer Letters. 2008; 270: 1-9. PubMed: https://pubmed.ncbi.nlm.nih.gov/18467025/ 
12. Hardell L, Carlberg M. Response to Ahlbom et al. Comments on Hardell and Carlberg Increasing Rates of Brian Tumors in the Swedish National Inpatient Register and the Causes of Death Register. Int. J. Environ. Res. Public Health 2015, 12, 3793-3813.

13. Comments on ROS and P53 gene mutations. P53 non-ionizing radiation and heat linked specifically to p53 mutation involved in astrocytoma).

14. Liu J, Du J, Zhang Y, Sun W, Smith BJ, et al. Redox Imbalance in the Development of Colorectal Cancer. J Cancer. 2017; 8:1586-1597. PubMed: https://www.ncbi.nlm.nih.gov/pmc/articles/PMC5535714/

15. Gago-Dominguez M, Castelao JE, Lipid peroxidation and renal cell carcinoma: Further supportive evidence and new mechanistic insights. Free Radical Biology \& Medicine. 2005; 40: 721-733. PubMed: https://pubmed.ncbi.nlm.nih.gov/16458203/

16. Mufti SI, Nachiappan V, Eskelson CD. Ethanol-mediated promotion of oesophageal carcinogenesis: association with lipid peroxidation and changes in phospholipid fatty acid profile of the target tissue. Alcohol and Alcoholism. 1997; 32:221-231.

17. Martin OCB, Naud N, Taché S, Debrauwer L, Chevolleau S, et al. Targeting Colon Luminal Lipid Peroxidation Limits Colon Carcinogenesis Associated with Red Meat Consumption. Cancer Prev Res (Phila). 2018; 11:569-580.

PubMed: https://pubmed.ncbi.nlm.nih.gov/29954759/

18. Hu W, Feng Z, Eveleigh J, Iyer G, Pan J, et al. The major lipid peroxidation product, trans- 4-hydroxy-2-nonenal, preferentially forms DNA adducts at codon 249 of human p53 gene, a unique mutational hotspot in hepatocellular carcinoma, Carcinogenesis. 2002; 23: 1781-1789.

PubMed: https://pubmed.ncbi.nlm.nih.gov/12419825/

19. Zhuo-Lin D, Ma Y. Aflatoxin sufferer and p53 gene mutation in hepatocellular carcinoma. World J Gastroenterol. 1998; 4: 28-29. PubMed: https://pubmed.ncbi.nlm.nih.gov/11819223/

20. Zhang J, Orang' O, Tonui P, Tong Y, Maina T, et al. Detection and Concentration of Plasma Aflatoxin Is Associated With Detection of Oncogenic Human Papillomavirus in Kenyan Women. Open Forum Infectious Diseases. 2019; 6: ofz354.

PubMed: https://pubmed.ncbi.nlm.nih.gov/31392332/

21. Skrzydlewska E, Sulkowski S, Koda M, Zalewski B, Kanczuga-Koda $\mathrm{L}$, et al.. Lipid peroxidation and antioxidant status in colorectal cancer. World J Gastroenterol. 2005; 11: 403-406.

PubMed: https://www.ncbi.nlm.nih.gov/pmc/articles/PMC4205348/

22. Jinchul K, Lili Y, Xuemei F, Yang X. WTP53 protein known as PUMA (acronym for "p53 upregulated modulator of apoptosis" caused switch from OP to glycolysis ie Warburg transition). UCLA. 2019

23. Kathrin $S$, Keller $S$, Wolter FE, Röglin L, Beil W, et al. Proximicins $A$ $B$, and C-Antitumor Furan Analogues of Netropsin from the Marine Actinomycete Verrucosispora Induce Upregulation of p5 3 and the Cyclin Kinase Inhibitor p21* Angew. Chem Int Ed. 2008; 47: 3258 -3261. PubMed: https://pubmed.ncbi.nlm.nih.gov/18348121/

24. Mantovani F, Collavin L. Del Sal G. Mutant p53 as a guardian of the cancer cell. Cell Death Differ. 2019; 26: 199-212.

PubMed: https://pubmed.ncbi.nlm.nih.gov/30538286/

25. Liang Y, Liu J, Feng Z. The regulation of cellular metabolism by tumor suppressor p53. Cell \& Bioscience. 2013; 3 .

PubMed: https://pubmed.ncbi.nlm.nih.gov/23388203/

26. Bhaskaran N, Quigley C, Paw C, Butala S, Schneider E, et al. Role of Short Chain Fatty Acids in Controlling Tregs and Immunopathology During Mucosal Infection. Front Microbiol. 2018; 9:1995.

PubMed: https://pubmed.ncbi.nlm.nih.gov/30197637/

27. IARC. Biological Agents. 2009; 100.

28. Habbous S, Chu KP, Lau H, Schorr M, Belayneh M, et al. Human papillomavirus in oropharyngeal cancer in Canada: analysis of 5 comprehensive cancer centres using multiple imputation. CMAJ: Canadian Medical Association J. 189: E1030-E1040.

PubMed: https://www.ncbi.nlm.nih.gov/pmc/articles/PMC5555753/
29. Mégraud F. H pylori antibiotic resistance: prevalence, importance, and advances in testing. Gut. 2004; 53: 1374-1384.

PubMed: https://www.ncbi.nlm.nih.gov/pmc/articles/PMC1774187/

30. Kohanski MA, Tharakan A, Lane AP, Ramanathan M Jr. Bactericidal antibiotics promote reactive oxygen species formation and inflammation in human sinonasal epithelial cells. Int Forum Allergy Rhinol. 2016; 6 : 191-200

PubMed: https://pubmed.ncbi.nlm.nih.gov/26624249/

31. Ben B, MD et al. Antibiotics increased risk of cancer. Eur J Cancer. 2015; 51: 2655-2664. https://pubmed.ncbi.nlm.nih.gov/26338196/

32. Annamari K, Harri R, Timo K, Eero P, Markku H, et al. Antibiotic predicts an increased risk of cancer. Wiley-Liss, Inc. 2008; 123: 2152-2155. PubMed: https://pubmed.ncbi.nlm.nih.gov/18704945/

33. Tim N. Antibiotics may increase the risk of bowel cancer. Medical News Today. 2017

34. Sigma M. Penicillin/Streptomycin/Amphotericin B Solution (100X), Tissue Culture Grade - Calbiochem-harmful and teratogenic and carcinogenic.

35. Velicer Christine M, Heckbert SR, Lampe JW. Antibiotic Use in Relation to the Risk of Breast Cancer. JAMA. 2004; 291: 827-835. PubMed: https://pubmed.ncbi.nlm.nih.gov/14970061/

36. Fraser Gary E, Jaceldo-Siegl K, Orlich M, Mashchak A, Sirirat R, et al. Dairy, soy, and risk of breast cancer: those confounded milks. Int $J$ Epidemiol. 2020; dyaa007.

PubMed: https://pubmed.ncbi.nlm.nih.gov/32095830/

37. IARC. Ampicillin was tested for carcinogenicity by oral administration in mice and rats. It increased the incidences of mononuclear-cell leukemia and of phaeochromocytomas of the adrenal medulla in male rats. A slight increase in the incidence of benign lung tumours was observed in female mice. 1990; 50.

38. Jyrkkanen J. Pesticide Carcinogens in Mothers' Milk and Total Diet; An Issue of Motherhood; ... Key Words: breast cancer, carcinogens, childhood cancers. 2010.

PubMed: https://jorma-jyrkkanen.livejournal.com/142897.html

39. Siegel RL, Torre LA, Soerjomataram I, Global patterns and trends in colorectal cancer incidence in young adults. Gut. 2019; 68: 2179-2185. PubMed: https://pubmed.ncbi.nlm.nih.gov/31488504/

40. Zhao J, Zhu Y, Wang PP, West R, Buehler S, et al. Interaction between alcohol drinking and obesity in relation to colorectal cancer risk: a casecontrol study in Newfoundland and Labrador, Canada. BMC Public Health. 2012; 12: 94.

PubMed: https://pubmed.ncbi.nlm.nih.gov/22296784/

41. Taniue K, Hayashi T, Kamoshida Y, Kurimoto A, Takeda Y, et al. UHRF1-KAT7-mediated regulation of TUSC3 expression via histone methylation/acetylation is critical for the proliferation of colon cancer cells. Oncogene. 2020; 39: 1018-1030.

42. Wang $X$, Moraes CT. Increases in mitochondrial biogenesis impair carcinogenesis at multiple levels. Molecuar Oncology. 2011; 399-409. PubMed: https://pubmed.ncbi.nIm.nih.gov/21855427/

43. Chen T. et al. (My comment) Pesticides induced mitochondrial fragmentation at low levels. Int J Mol Sci. 2017; 18. PubMed: https://www.ncbi.nlm.nih.gov/pmc/articles/PMC5751110/

44. Alavanja MCR, Bonner MR. Occupational pesticide exposures and cancer risk. A review. J Toxicol Environ Health B Crit Rev. 2018; 15: 238-263.

PubMed: https://pubmed.ncbi.nlm.nih.gov/22571220/

45. Ray WA, Stein M, May D. Common antibiotic found to pose increased heart risk. New Engl J Med. 2012.

PubMed: https://www.ncbi.nlm.nih.gov/pmc/articles/PMC3374857/

46. Giudicessi John R, Ackerman MJ, Azithromycin and Risk of Sudden Cardiac Death: Guilty as Charged or Falsely Accused? Cleve Clin J 
Med. 2013; 80: 539-544.

PubMed: https://pubmed.ncbi.nlm.nih.gov/24001961/

47. University of British Columbia. Commonly used antibiotics may lead to heart problems." Science Daily. 2019.

www.sciencedaily.com/releases/2019/09/190910154710.htm

48. FDA. FDA warns some pesticides can cause fatal heart damage. NBC Health News. 2018.

49. Arias PL, Esteller M. Coding and Non-Coding Genes Silenced Epigenetically in Cancer. Open Biol. 2017; 7: PMC5627056.

50. Sumei W, et al. Mutual regulation of microRNAs and DNA methylation in human cancers. Epigenetics. 2017; 12: 187-197.

PubMed: https://pubmed.ncbi.nlm.nih.gov/28059592

51. Rivlin N, Brosh R, Oren M, Rotter V. Mutations in the p53 Tumor Suppressor Gene: Important Milestones at the Various Steps of Tumorigenesis. Genes \& cancer. 2011; 2: 466-474.

PubMed: https://pubmed.ncbi.nlm.nih.gov/21779514/
52. Cadernos de Saúde Pública. Brazil Tribal Cancers. Print version ISSN 0102-311XOn-line version ISSN 1678-4464Cad. Saúde Pública. 2019; 35.

53. Raturi A, Simmen T. Where the endoplasmic reticulum and the mitochondrion tie the knot: The mitochondria-associated membrane (MAM) Biochemica et Biophysica Acta (BBA) - Molecular Cell Res. 2013; 1833: 213-224.

PubMed: https://pubmed.ncbi.nlm.nih.gov/22575682/

54. Wallace DC. Mitochondria and cancer. Nat Rev Cancer. 2012; 12 : 685-698.

PubMed: https://pubmed.ncbi.nlm.nih.gov/23001348/

55. Baxter JS, Leavy OC, Dryden NH. et al. Capture Hi-C identifies putative target genes at 33 breast cancer risk loci. Nat Commun. 2018; 9: 1028. PubMed: https://pubmed.ncbi.nlm.nih.gov/29531215/

56. Steinberg P. Red meat derived Nitroso Compounds, Lipid Peroxidation Products and Colorectal Cancer. Foods. 2019. 8: 252.

PubMed: https://pubmed.ncbi.nlm.nih.gov/31336781/ 